\title{
Turbulent kinetic energy in the right ventricle: Potential MR marker for risk stratification of adults with repaired Tetralogy of Fallot
}

Alexandru Grigorescu Fredriksson, Aleksandra Trzebiatowska-Krzynska, Petter Dyverfeldt, J an Engvall, Tino Ebbers and Carljohan Carlhäll

The self-archived postprint version of this journal article is available at Linköping University Institutional Repository (DiVA):

http:/ / urn.kb.se/ resolve?urn=urn:nbn:se:liu:diva-143780

N.B.: When citing this work, cite the original publication.

Fredriksson, A. G., Trzebiatowska-Krzynska, A., Dyverfeldt, P., Engvall, J ., Ebbers, T., Carlhäll, C., (2017), Turbulent kinetic energy in the right ventricle: Potential MR marker for risk stratification of adults with repaired Tetralogy of Fallot, J ournal of Magnetic Resonance Imaging.

https:// doi.org/ 10.1002/jmri.25830

Original publication available at:

https:// doi.org/ 10.1002/jmri.25830

Copyright: Wiley

http:/ / eu.wiley.com/WileyCDA/ 
Turbulent kinetic energy in the right ventricle: Potential MR marker for risk stratification of adults with repaired Tetralogy of Fallot 


\section{Abstract}

\section{Background}

The number of adult patients with long-term complications after repair of Tetralogy of Fallot (ToF) is increasing. Pulmonary regurgitation (PR) leads to right ventricular (RV) remodeling and dysfunction. PR causes turbulent flow within the RV, which may contribute to RV remodeling. 4D flow MR permits quantification of intracardiac turbulent kinetic energy (TKE).

\section{Purpose}

To assess RV TKE in patients with repaired ToF (rToF) and a spectrum of PR, as well as to investigate the relationship between these 4D flow markers and RV remodeling.

\section{Study type}

Prospective cohort study.

\section{Subjects}

17 patients with rToF and 10 healthy controls. Patients were divided into two groups based on PR fraction, one lower PR fraction group ( $\leq 11 \%)$ and one higher PR fraction group $(>11 \%)$.

\section{Field strength/sequences}

$3 \mathrm{D}$ cine phase contrast (4D flow), 2D cine phase contrast (2D flow) and bSSFP at 1.5T.

\section{Assessment}

The RV volume was segmented in the morphologic short-axis images and TKE parameters were computed inside the segmented RV volume throughout diastole. 


\section{Statistical tests}

One-way ANOVA with Bonferroni post-hoc test; Unpaired t-test; Pearson correlation coefficients; Simple and stepwise multiple regression models; Intra-class correlation coefficient (ICC).

\section{Results}

The higher PR fraction group had more remodeled RVs (140 \pm 25 vs. $107 \pm 22$ (lower PR fraction, $\mathrm{p}<0.01$ ) and $93 \pm 15 \mathrm{ml} / \mathrm{m}^{2}$ (healthy, $\mathrm{p}<0.001$ ) for $\mathrm{RV}$ end diastolic volume index (RVEDVI)) and higher TKE values (5.95 \pm 3.15 vs. $2.23 \pm 0.81$ (lower PR fraction, $\mathrm{p}<0.01$ ) and $1.91 \pm 0.78 \mathrm{~mJ}$ (healthy, $\mathrm{p}<0.001$ ) for Peak Total $R V T K E$ ). Multiple regression analysis between RVEDVI and 4D/2D flow parameters showed that Peak Total RV TKE was the strongest predictor of RVEDVI $\left(\mathrm{R}^{2}=0.47, \mathrm{p}=0.002\right)$.

\section{Data conclusion}

The 4D flow specific TKE markers showed a slightly stronger association with RV remodeling than conventional 2D flow PR-parameters. These results propose novel hemodynamic aspects of PR in the development of late complications after ToF repair.

\section{Keywords}

4D flow, MRI, Turbulence, Tetralogy of Fallot, Turbulent kinetic energy

\section{Introduction}

Tetralogy of Fallot (ToF) is the most common cyanotic congenital heart disease (1). With advances in diagnosis and treatment, post-repair survival has markedly improved, with a large 
increase in the number of patients reaching adulthood with repaired ToF (rToF) (2). Consequently, new clinical challenges associated with the long-term complications of early repair have emerged. Among these later complications are pulmonary valve incompetence and subsequently varying degrees of pulmonary regurgitation (PR) $(3,4)$.

Initially considered unimportant, in the last decades several studies have shown a clear relation between PR and many late complications. The chronic right ventricular (RV) volume overload following the PR is considered central in the development of progressive right ventricular (RV) remodeling, fibrosis and subsequently RV failure (5-7). PR and chronic volume overload are usually well tolerated for many years, however, left uncorrected irreversible ventricular remodeling will occur $(5,7,8)$.

Pulmonary valve replacement (PVR) has shown beneficial effects in rToF patients with PR (7-10). With time, many patients with surgical ToF repair are reaching a point where the need of PVR will be imminent. The timing of this procedure is often unclear due to the variable and suboptimal prognosis of these adult patients (4, 7-11).

Despite identification of PR as an important driving force in the development of these late complications, the hemodynamic alterations, other than the volume overload brought on by the PR, are not fully explored. Disturbed or turbulent flow is rare in the normal cardiovascular system and is known to damage cardiovascular tissues $(12,13)$. Increased amounts of turbulent kinetic energy (TKE), a measure of the degree of turbulent flow, have been demonstrated with cardiac pathology such as valvular insufficiency (14). Studies have also shown an association between flow disturbances and dilation/remodeling of the ascending aorta in patients with aortic valve stenosis (15). Accordingly, turbulent flow may contribute to 
the development of RV remodeling, right ventricular outflow tract fibrosis and other complications seen in patients with rToF and significant PR.

Magnetic resonance imaging (MRI) has emerged as a powerful tool for the assessment of ToF patients (16). Time-resolved, three-dimensional, three-directional phase-contrast (4D flow) MRI permits not only visualization and quantification of the blood flow within the heart (1719), but also of intracardiac turbulent kinetic energy (TKE) $(14,20)$.

In this study, we sought to assess RV TKE in adult patients with rToF and varying degrees of PR as well as in healthy controls. Specifically, we wanted to investigate the relationship between these novel 4D flow markers and RV remodeling in order to evaluate their potential usefulness in patient risk stratification.

\section{Materials and methods}

\section{Study population}

A total of 27 patients with diagnosis of ToF that had undergone surgical repair were prospectively enrolled from outpatients at the Department of Cardiology, Linköping University Hospital. In addition, ten healthy age-matched controls with no history of cardiac disease or cardiac medication were enrolled. The study was approved by the regional Ethical Review Board in Linköping, Sweden and all participants gave written informed consent.

Exclusion criteria for all study subjects were contraindications for MRI examination. Exclusion criteria for the patients were more than mild tricuspid valvular disorder and previous PVR with no present PR. In total 10 patients were excluded, please see Results for 
details. Five patients had previously undergone PVR, where only one was included because of persistent PR after the operation.

The patient group was further divided into two strata for comparative purposes based on PR fraction computed from conventional 2D flow MRI data using the patient group median of $11 \%$ as cut-off (median 11\%, range $1-50 \%$ ): one group with lower PR fraction $(\leq 11 \%$, $\mathrm{N}=9$; 6 male, 3 female) and one group with higher PR fraction ( $>11 \%, N=8$; 3 male, 5 female) (Table 1).

\section{Data acquisition}

4D flow and 2D flow MRI data as well as morphological short-axis and apical long-axis cine images were acquired using a clinical 1.5 T MRI scanner (Philips Achieva; Philips Medical Systems, Best, the Netherlands). The 4D flow data were acquired during free breathing using retrospectively ECG-gated and navigator-gated three-dimensional, three-directional phase contrast MRI (4D flow MRI) with asymmetric 4-point motion-encoding and either Cartesian or a stack of spiral readouts, as previously described $(20,21)$. The Cartesian sequence scan parameters included: Velocity encoding range $(\mathrm{VENC})=100 \mathrm{~cm} / \mathrm{s}$, flip angle $8^{\circ}$, echo time $=$ $3.7 \mathrm{~ms}$, repetition time $=6.3 \mathrm{~ms}$, parallel imaging $($ SENSE) speed-up factor $=2$, field of view $($ FOV $)=230 \times 230-274 \times 274 \mathrm{~mm}^{2}$ with a spatial resolution of $3 \mathrm{~mm}$ isotropic and an acquired temporal resolution of 49.2 ms. The spiral sequence scan parameters included: VENC $=120 \mathrm{~cm} / \mathrm{s}$, flip angle $8^{\circ}$, echo time $=3.7 \mathrm{~ms}$, repetition time $=12 \mathrm{~ms}$, spiral interleaves $=10, \mathrm{FOV}=280 \times 280 \mathrm{~mm}^{2}$ with a reconstructed spatial resolution of $2.8 \mathrm{~mm}$ isotropic and an acquired temporal resolution of approximately $48 \mathrm{~ms}$. A five-element cardiac SENSE coil was used for both protocols. Total scan time including navigator efficiency was approximately 15-20 min. 
Mean velocity fields used were reconstructed from the 4D Flow MRI data using conventional phase-difference algorithms. Velocity data were corrected for concomitant gradient field effects on the scanner and for phase-wraps and background phase-offsets using methods previously described $(22,23)$. The magnitude images of the individual flow-encoding segments were reconstructed to compute the TKE. The TKE is defined as:

$$
T K E=\frac{1}{2} \rho \sum_{i=1}^{3} \sigma_{i}^{2}\left[J / m^{3}\right]
$$

where $\rho$ is the fluid density, and $\sigma_{i}$ is the velocity fluctuation intensity in three orthogonal directions. For the asymmetric four-point flow encoding, as used here, $\sigma_{i}$ is obtained as (24):

$$
\sigma_{i}=\frac{1}{k_{v}} \sqrt{2 \frac{|S|}{\left|S_{i}\right|}}
$$

where $\left|S_{i}\right|$ and $|S|$ are the magnitude of MR signal with and without motion sensitivity encoding, respectively. $\mathrm{k}_{\mathrm{v}}(=\pi / V E N C)$ describes the motion sensitivity.

The bSSFP cine images were acquired in 30 time frames during end-expiratory breath holds. Acquired pixel size was $1.67 \times 1.67-1.78 \mathrm{~mm}$ for the long-axis images and 2-2.19 x 1.78-2 $\mathrm{mm}$ for the short-axis images. The short-axis stack had a slice thickness of $8 \mathrm{~mm}$. 2D flow data were acquired in planes perpendicular to the proximal ascending aorta and pulmonary trunk. The acquisition was retrospectively ECG-gated using the following parameters: slice thickness $8 \mathrm{~mm}$, acquired pixel size 2.5 x $2.5 \mathrm{~mm}$ (reconstructed to 1.25 x $1.25 \mathrm{~mm}$ ), FOV of $320 \times 260 \mathrm{~mm}$, SENSE factor $=2$, VENC $=200 \mathrm{~cm} / \mathrm{s}$, repetition time $=4.6 \mathrm{~ms}$ and echo time $=2.7 \mathrm{~ms}$, TFE-factor 4 with a temporal resolution of $36.9 \mathrm{~ms}$. Furthermore, all patients had a 12-leads resting ECG taken for assessment of QRS-duration.

\section{Data analysis}


Following previous work on intracardiac TKE $(14,25)$, the TKE in the RV was computed for each diastolic timeframe by integrating the TKE within the RV volume. The analysis was performed by one investigator (AF) with 7 years of experience in cardiovascular MR. For those purposes, the RV volume was segmented in the morphologic short axis images using the free research segmentation software Segment v 1.9 R4040 (http://segment.heiberg.se) (26). End-diastolic (ED) and end-systolic (ES) time points were visually identified in the four-chamber images as the first timeframe in which the tricuspid valve was closed after diastolic filling and the last timeframe before tricuspid valve opening after systolic ejection, respectively. All diastolic timeframes between ED and ES were segmented with guidance of the long-axis images following the compact myocardial contour. Segmentation was performed to encompass the whole right ventricular outflow tract (RVOT) and a small part of the proximal pulmonary trunk to ensure that the pulmonary valve was included in the segmentation. No turbulence was expected to occur in the pulmonary arteries during diastole. The segmentation was temporally and spatially resampled to match the resolution of the 4D flow data and was manually registered to the 4D flow data using an in-house MatLab script in order to eliminate effects of patient movements during the scan. The final segmentation was used in the subsequent analysis of TKE (14).

Several TKE-specific markers were formulated in order to describe the hemodynamic effects of the PR. For every timeframe, the TKE inside the 3D RV volume was integrated spatially to obtain the total amount of RV TKE in the respective timeframe. The RV TKE in the diastolic timeframe with highest TKE was defined as Peak Total RV TKE. The total amount of RV TKE over diastole was divided with the number of diastolic timeframes in order to determine the average TKE inside the segmented RV volume over diastole, Average RV TKE. Further, the maximum local TKE was plotted over diastole and the highest value was measured, Peak 
$R V T K E$. This was performed after median filtering to minimize the effects of noise on the identification of Peak RV TKE. Anatomic localization and the time of occurrence of the Peak $R V$ TKE was assessed by visually showing the respective voxel as a dot in the TKEvisualizations (Figure 1).

For the clinical RV volumes, a second RV segmentation was performed using a clinical segmentation protocol. In the 2D flow data, the proximal ascending aorta and the pulmonary trunk were delineated in all timeframes over the cardiac cycle and forward and backward flow were computed in order to assess PR volumes and fractions.

\section{D flow visualization}

Commercially available flow visualization software (EnSight, CEI, Apex, NC) was used to visualize TKE within the RV throughout diastole. TKE was visually inspected for significant artifacts. The blood flow patterns were visualized as streamlines together with the TKE visualization throughout diastole to give a brief overview of some flow events associated with the TKE development (Figure 1). All visual inspections were performed by two investigators (AF and CJC) in consensus. The investigators had 7 and 12 years of experience in cardiovascular MR, respectively.

\section{Inter- and intra-observer variability}

The method was evaluated for inter- and intra-observer variability. Two investigators (AF and ATK, Inv 1 and Inv 2, respectively) carried out the analysis in six randomly selected datasets: two healthy controls, two subjects with lower PR and two subjects with higher PR. Inv 1 had substantial experience and Inv 2 had moderate experience of segmenting the RV from bSSFP short axis images. Further, in order to assess the intra-observer variability, one investigator 
(AF, Inv 1) repeated the analysis in all six subjects approximately six months after the first analysis. The first analysis performed by Inv 1 is referred to as Analysis 1 (A1) and the second as Analysis 2 (A2).

\section{Statistical analysis}

Results were assessed for normality by computation of parameter residuals and creation of normality dot plots. This was done due to the small study group sizes, and all parameters presented satisfactory normality, rendering parametric statistical tests adequate. All results are given as mean $\pm \mathrm{SD}$ (range) unless otherwise stated. The statistical significance level was set at $\mathrm{p}<0.05$. For inter-group comparisons, a one-way analysis of variance (ANOVA) with a Bonferroni post-hoc test was used. Unpaired t-test analyses were performed for parameters present for only two sub-groups (QRS-duration and time from ToF repair). Measures of PR volume and PR fraction were not available in one subject due to technical issues, thus analyses involving these data were performed for 26 subjects.

Correlations were performed using Pearson correlation coefficients. Simple regression and stepwise multiple regression models were used to assess parameters as predictors of RVEDVI. Repeated simple regression analyses were initially performed to determine which parameters should be included in the multiple model as independent predictors (Table 3). A multiple regression model was constructed, and based upon the simple regression analyses, the predictor parameters chosen were PR volume and PR fraction, Average RV TKE, Peak Total $R V$ TKE and Peak $R V$ TKE. For each step, the least significant parameters were excluded from the model. 
Inter- and intra-observer variability was assessed using intra-class correlation coefficient (ICC) for the TKE markers Average RV TKE, Peak Total RV TKE and Peak RV TKE. ICC estimates and their 95\% confidence intervals (CI) were based on a single-measure absolute agreement 2-way random-effects model (ICC $(2,1)$ ) for the inter-observer variability tests and a single-measure absolute agreement 2-way mixed-effects model (ICC $(3,1)$ ) for the intraobserver variability test (27). Statistical analyses were performed using the statistical softwares Statistica v.10 (StatSoft, Tulsa, OK) and IBM SPSS v. 24 (IBM Corp, Armonk, NY).

\section{Results}

Out of the 27 rToF patients enrolled, five patients had previously undergone PVR, where only one was included because of persistent PR after the operation. Six patients were excluded because of technical issues in their datasets. Consequently, 17 patients with rToF (9 male, 8 female) and 10 healthy subjects were included in the final study.

\section{Basic clinical parameters}

The patient sub-groups and the healthy control group presented no significant differences in any of the basic clinical parameters, namely age, BSA, heart rate or blood pressure (Table 1). No significant difference was present between the patient sub-groups with respect to QRSduration $(\mathrm{p}=0.099)$ or in time from ToF repair $(\mathrm{p}=0.169)$. The group with higher PR fraction presented higher RV remodeling indices than both the group with lower PR fraction and the healthy control group ( $\mathrm{p}<0.01, \mathrm{p}<0.001$ for RVEDVI, respectively, and $\mathrm{p}<0.001$, $\mathrm{p}<0.001$ for RVESVI, respectively). The group with lower PR fraction also presented higher RVESVI than the healthy control group $(\mathrm{p}=0.005)$. Both patient sub-groups had lower RVEF than the healthy control group ( $<0.001$ for both) (Table 1). 


\section{D flow parameters}

As expected, the group with higher PR fraction had also larger PR volumes than both the group with lower PR fraction and the healthy control group $(\mathrm{p}<0.001$, $\mathrm{p}<0.001$ for PR fraction, respectively, and $\mathrm{p}<0.001, \mathrm{p}<0.001$ for PR volume, respectively) (Table 2). Conservation of mass was assessed for the 2D flow parameters by computing the difference between the net forward flow in the ascending aorta and the net forward flow in the pulmonary trunk, and no significant loss of mass was found $(82 \pm 22 \mathrm{ml}$ and $84 \pm 20 \mathrm{ml}$, respectively, $\mathrm{p}=0.189$ ).

\section{D flow parameters}

The group with higher PR fraction had significantly higher amounts of diastolic RV TKE than both the group with lower PR fraction and the healthy control group, reflected by higher values of Average RV TKE ( $\mathrm{p}=0.002$ and $\mathrm{p}<0.001$, respectively), Peak Total RV TKE ( $\mathrm{p}=$ 0.001 and $\mathrm{p}<0.001$, respectively) (Figure 2, A through C) and Peak RV TKE (p $<0.001$ and $\mathrm{p}<0.001$, respectively) (Table 2).

\section{TKE visualization}

The substantially larger TKE development in the higher PR fraction group was located in the RVOT during the early diastolic filling phase, reaching centrally and apically inside the RV chamber as a plume around the regurgitant jet (Figure 1). Peak RV TKE was consistently situated in the RVOT in all patients in the higher PR fraction group. None of the healthy subjects presented locally elevated TKE in the RVOT. Contrary to the higher PR fraction patients, the measured Peak RV TKE was in the majority of healthy subjects situated in the RV inflow region during diastolic filling. This TKE pattern was also observed in the patients 
of the lower PR fraction group. Three patients in this group had TKE development in the RVOT; these patients also presented the highest PR fractions in this group, between 4 and 11\%. Peak RV TKE values in these three patients were higher (ranging from 176 to 246 $\mathrm{mJ} / \mathrm{m}^{3}$ ) than those presented by the healthy control group.

\section{Correlation and regression analyses}

PR fraction was related to the TKE-parameters and Peak RV TKE correlated the strongest $(\mathrm{r}=$ 0.87), followed by Peak Total RV TKE ( $\mathrm{r}=0.85)$ and Average RV TKE $(\mathrm{r}=0.80)(\mathrm{p}<0.05$ for all). The simple regression analyses performed showed that none of the basic clinical parameters were significant predictors of RVEDVI with low $\mathrm{R}^{2}$-values and non-significant $\mathrm{p}$ values (Table 3, first column), while all conventional 2D flow parameters, as well as the 4D flow parameters were moderately predictive of RVEDVI at high significance levels. Peak Total $R V$ TKE was the strongest predictor of RVEDVI in the simple regression analysis among the tested variables $\left(\mathrm{R}^{2}=0.47, \mathrm{p}=0.002\right)$, followed by Average $R V T K E\left(\mathrm{R}^{2}=0.44, \mathrm{p}\right.$ $=0.004)$ and $P R$ volume $\left(R^{2}=0.37, p=0.009\right)$. These significant parameters were included in the subsequent backward stepwise multiple regression analysis (Table 3, second column). All models were significant at $\mathrm{p}<0.05$, although no parameter significance was reached until only Peak Total $R V$ TKE remained at $\mathrm{R}^{2}=0.47(\mathrm{p}=0.002)$ with an adjusted $\mathrm{R}^{2}=0.44(\mathrm{p}=$ 0.002) for the model. Throughout the analysis, the adjusted $\mathrm{R}^{2}$-values increased (Table 3 , bottom section) (Figure 3).

\section{Inter- and intra-observer variability}

Excellent degrees of reliability were found for the inter-observer analysis between Inv 1 and Inv 2. The single measure ICC $(2,1)$ was $0.986(95 \%$ CI of $0.917-0.998, \mathrm{p}<0.001)$ for Average RV TKE, 0.984 (95\% CI of $0.898-0.998, \mathrm{p}<0.001)$ for Peak Total RV TKE and 
0.995 (95\% CI of $0.973-0.999, \mathrm{p}<0.001)$ for Peak RV TKE. Further, excellent degrees of reliability were found for the intra-observer analysis between Inv 1 A1 and Inv 1 A2. The single measure ICC $(3,1)$ was $0.998(95 \%$ CI of $0.985-1.000, \mathrm{p}<0.001)$ for Average $R V$ TKE, 0.995 (95\% CI of 0.971 - 0.999, p < 0.001) for Peak Total RV TKE and 0.997 (95\% CI of $0.981-1.000, \mathrm{p}<0.001$ ) for Peak RV TKE.

\section{Discussion}

In the present study, TKE in the RV of patients with rToF and a spectrum of PR was investigated using 4D flow MRI. TKE was increased in the rToF patients with PR compared to healthy controls, and related slightly stronger to indices of RV remodeling than conventional 2D flow based parameters of PR. The highest TKE values were mainly located in the RV outflow tract.

Both patient sub-groups and the healthy control group were well matched with no significant differences in basic clinical parameters, such as heart rate, that could affect TKE measurements. There was no significant difference in QRS-duration between the two patient groups, even though the higher PR fraction group presented higher RVEDVI, which previously has been linked to increased QRS-durations (28). The mean QRS-durations were well under the threshold of $>180$ ms proposed for increased risk for arrhythmias (29) but higher than normal ranges in both patient groups. Similarly, the mean RVEDVI values of both patient subgroups were below the proposed threshold for irreversible changes and did not meet the PVR-indications (7, 8, 10, 29-31). The mean RVEF in the higher PR fraction group was however lower than the limits proposed for reintervention (7). In accordance to the many present guideline documents and meta-analyses, significant PR present in combination with parameters of RV deterioration or symptoms constitute indication for PVR (7, 8, 10, 29- 
31). The higher PR fraction subgroup thereby falls right on the threshold for reintervention. Markedly increased levels of TKE could be seen in the RVs of this higher PR fraction group, both globally (Average RV TKE, Peak Total RV TKE) and locally (Peak RV TKE), even before clear indication for clinical reintervention is present.

The visualization of TKE inside the RV volume of these higher PR fraction patients showed that the development of TKE and the Peak RV TKE values were located in the outflow tract at the time of early RV diastole, coinciding with the occurrence of PR (Figure 1, movie in Supporting file 2). The similar patterns regarding anatomic locale and time of occurrence for the PR and TKE development in the higher PR fraction patients proposes that TKE reflects the severity of the regurgitation, as previously shown for mitral regurgitation (14). In the mitral regurgitation study, the left atrial (LA) Peak Total TKE reached levels between 13 and $37 \mathrm{~mJ}$, which is substantially higher than the highest levels observed in the present study (5.95 $\pm 3.15 \mathrm{~mJ}$ in the higher PR fraction group). This would be expected, as pressure gradients between the left ventricle $(\mathrm{LV})$ and LA in the setting of mitral regurgitation during systole widely exceeds the gradients present between the pulmonary artery and the RV during diastole. However, the mechanism behind the development of elevated TKE would be similar.

No statistically significant difference in TKE levels were seen between the healthy controls and the lower PR fraction group, however the higher PR fraction group presented overall higher levels of TKE than the other two groups. A recent study by Jeong et al. showed that the RV kinetic energy (KE) corrected for body surface area was slightly higher in a cohort of rToF patients with PR compared to healthy volunteers whereas no significant intergroup difference in pulmonary flow (RV stroke volume) was observed, reflecting a higher energy loss in the RVs of patients with rToF without the ability to generate increased flow (32). 
Further, Jeong et al. found that the highest KE was seen in the RVOT during both systole and diastole, due to the presence of PR. These finding mirror the findings of the current study, where progressive increase in PR fraction brought about not only progressively higher local Peak $R V$ TKE values, but also an increase in Total Peak RV TKE and Average RV TKE, reflecting a higher energy loss inside the entire RV throughout diastole. This could in turn explain why Peak Total $R V T K E$ and Average $R V$ TKE were stronger predictors of higher RVEDVI than was Peak RV TKE, as shown by the simple regression models.

The multiple regression model showed that the strongest independent predictor variable for RVEDVI was the 4D flow specific parameter Peak Total RV TKE. The correlations between the conventional 2D flow parameters and the 4D flow specific TKE parameters were high. These parameters might still not be fully interchangeable, as the $4 \mathrm{D}$ flow specific TKE parameters reflect not only the amount of PR but also flow specific effects of the PR.

In a recent study, Hirtler and colleagues studied right heart vorticity in repaired rToF patients and healthy volunteers and found significant correlations between pulmonary regurgitant flow and the occurrence of vorticity in the right heart (33). The authors conclude that changes in vorticity may be a cause for ventricular energy loss and progressive RV dilatation. Interestingly, in the patients they also found a negative correlation between right heart vorticity and RVEDVI, which is in contrast to the positive correlation we observed between RV TKE and RVEDVI. The authors speculate that the negative correlation may be explained by the technical note that vorticity is the circulation of fluid per unit area. Hence, if a small RV has similar amounts of circulation as a large $\mathrm{RV}$, the smaller one will have a higher value of circulation per unit area i.e. vorticity will be higher despite lower RVEDVI. 
It is reasonable to believe that there are other factors besides the RV volume overload due to worsening PR that contribute to RV dilation. Transannular patching during initial repair can lead to aneurysmal development in the RVOT. This local remodeling will contribute to an increase in RVEDVI $(34,35)$, and it has been discussed that aneurysmal resection during PVR procedures could partly explain the postoperative reduction of RV volumes (36). A previous study however found that a significant portion of the included patients presented outflow aneurysms even though transannular patching was not initially performed, raising the question of other factors important for aneurysmal development and RV remodeling (35).

Further, evidence of fibrosis occurring in the RV outflow tract in patients with rToF has been presented in multiple studies (37-39), even in areas outside the surgical incision $(38,40)$. The impact of fibrosis on late complications has been discussed (41). Previous studies have demonstrated that disturbed flow is linked to endothelial dysfunction $(12,13)$, that could impact subendocardial structures causing fibrotization and remodeling, in this case presumably in the RVOT and adjacent ventricular segments. Moreover, the severity of PR has been related to an increase of the RV extracellular volume, synonymous with increased levels of diffuse RV myocardial fibrosis (42). In line with this, Munkhammar et al. did also find a positive correlation between PR severity, the degree of fibrosis as well as larger RV volumes (40).

The Peak Total RV TKE in the present cohort was the strongest predictor of RVEDVI, suggesting that elevated TKE in the outflow tract could act as a mediator of myocardial fibrosis, local aneurysms and increasing RVEDVI. This proposed mechanism of fibrotization and dilation can be further supported by the uniform occurrence of Peak RV TKE in the outflow tract during diastole in the patients with larger PR. 4D flow specific TKE parameters 
could thus reflect different pathophysiologic mechanisms of late RV remodeling in patients with rToF, integrating not only the PR size but also aspects of mechanotransduction. As the visualizations of TKE showed, the PR and development of TKE extended further down into the chamber than only the RVOT, which in turn could explain fibrotization of areas outside the region of surgical repair.

The present study has some limitations. The study was conducted on a relatively small number of subjects. A more clinical cut off value for separation of the two patient sub-groups based on PR would have been useful. However, the main findings in this study were based on the regression analyses, and to a lesser degree on the intergroup comparisons. Data were acquired at rest in subjects in the supine position with sinus rhythm. Data were acquired at end-expiration and the results may vary over the respiratory cycle. Patient motion during MRI scan may give rise to motion artifacts or data mismatch. Registration of data during the analysis aimed at alleviating this mismatch. TKE data may contain artifacts that may influence the measured values of especially Peak $R V T K E$, why median filtering of TKE was performed. For the integrative measurements, Peak Total RV TKE and Average RV TKE, occurrence of both negative and positive artifacts were considered to cancel each other out, and no filtering was performed for the calculation of these (43). In this cross-sectional study, RV TKE was the strongest predictor of RVEDVI, however we plan to analyze whether RV TKE can predict increased RVEDVI over time in future longitudinal studies.

In conclusion, TKE in the RV of patients with rToF and PR can be quantified and visualized using 4D flow MRI. TKE was elevated in rToF patients compared to healthy subjects, and related slightly stronger to indices of RV remodeling than conventional 2D flow based PRparameters. The highest values were mainly located in the RV outflow tract, which has been 
shown to be prone to fibrosis and aneurysmal development. These findings propose novel hemodynamic aspects of pathophysiological mechanisms of PR in the development of late complications after ToF repair, that has potential to serve as markers for risk stratification.

\section{References}

1. Benjamin EJ, Blaha MJ, Chiuve SE, Cushman M, Das SR, Deo R, de Ferranti SD, Floyd J, Fornage M, Gillespie C, et al: Heart Disease and Stroke Statistics-2017 Update: A Report From the American Heart Association. Circulation 2017, 135:e146-e603.

2. Apitz C, Webb GD, Redington AN: Tetralogy of Fallot. Lancet 2009, 374:14621471.

3. Monro JL: Transventricular Repair of Tetralogy of Fallot. Operative Techniques in Thoracic and Cardiovascular Surgery 2005, 10:45-53.

4. Guleserian KJ: Adult congenital heart disease: surgical advances and options. Progress in cardiovascular diseases 2011, 53:254-264.

5. Bouzas B, Kilner PJ, Gatzoulis Ma: Pulmonary regurgitation: not a benign lesion. European heart journal 2005, 26:433-439.

6. Davlouros Pa, Niwa K, Webb G, Gatzoulis Ma: The right ventricle in congenital heart disease. Heart (British Cardiac Society) 2006, 92 Suppl 1:i27-38.

7. Geva T: Indications and timing of pulmonary valve replacement after tetralogy of Fallot repair. Seminars in thoracic and cardiovascular surgery Pediatric cardiac surgery annual 2006:11-22.

8. Therrien J, Provost Y, Merchant N, Williams W, Colman J, Webb G: Optimal timing for pulmonary valve replacement in adults after tetralogy of Fallot repair. The American journal of cardiology 2005, 95:779-782. 
9. Burchill LJ, Wald RM, Harris L, Colman JM, Silversides CK: Pulmonary valve replacement in adults with repaired tetralogy of Fallot. Seminars in thoracic and cardiovascular surgery Pediatric cardiac surgery annual 2011, 14:92-97.

10. Cheung EW, Wong WH, Cheung YF: Meta-analysis of pulmonary valve replacement after operative repair of tetralogy of fallot. The American journal of cardiology 2010, 106:552-557.

11. Lindsey CW, Parks WJ, Kogon BE, Sallee D, 3rd, Mahle WT: Pulmonary valve replacement after tetralogy of Fallot repair in preadolescent patients. The Annals of thoracic surgery 2010, 89:147-151.

12. Davies PF, Remuzzi A, Gordon EJ, Dewey CF, Jr., Gimbrone MA, Jr.: Turbulent fluid shear stress induces vascular endothelial cell turnover in vitro. Proc Natl Acad Sci U S A 1986, 83:2114-2117.

13. Jenkins NT, Padilla J, Boyle LJ, Credeur DP, Laughlin MH, Fadel PJ: Disturbed blood flow acutely induces activation and apoptosis of the human vascular endothelium. Hypertension 2013, 61:615-621.

14. Dyverfeldt P, Kvitting JP, Carlhall CJ, Boano G, Sigfridsson A, Hermansson U, Bolger AF, Engvall J, Ebbers T: Hemodynamic aspects of mitral regurgitation assessed by generalized phase-contrast MRI. Journal of magnetic resonance imaging : JMRI 2011, 33:582-588.

15. Hope MD, Hope TA, Crook SE, Ordovas KG, Urbania TH, Alley MT, Higgins CB: 4D flow CMR in assessment of valve-related ascending aortic disease. JACC Cardiovascular imaging 2011, 4:781-787.

16. Kilner PJ: The role of cardiovascular magnetic resonance in adults with congenital heart disease. Progress in cardiovascular diseases 2011, 54:295-304. 
17. Geiger J, Markl M, Jung B, Grohmann J, Stiller B, Langer M, Arnold R: 4D-MR flow analysis in patients after repair for tetralogy of Fallot. European radiology 2011, 21:1651-1657.

18. Vasanawala SS, Hanneman K, Alley MT, Hsiao A: Congenital heart disease assessment with 4D flow MRI. Journal of magnetic resonance imaging : JMRI 2015, 42:870-886.

19. François CJ, Srinivasan S, Schiebler ML, Reeder SB, Niespodzany E, Landgraf BR, Wieben O, Frydrychowicz A: 4D cardiovascular magnetic resonance velocity mapping of alterations of right heart flow patterns and main pulmonary artery hemodynamics in tetralogy of Fallot. Journal of cardiovascular magnetic resonance : JCMR 2012, 14:16.

20. Dyverfeldt P, Kvitting J-PE, Sigfridsson A, Engvall J, Bolger AF, Ebbers T: Assessment of fluctuating velocities in disturbed cardiovascular blood flow: in vivo feasibility of generalized phase-contrast MRI. Journal of magnetic resonance imaging : JMRI 2008, 28:655-663.

21. Petersson S, Sigfridsson A, Dyverfeldt P, Carlhall CJ, Ebbers T: Retrospectively gated intracardiac 4D flow MRI using spiral trajectories. Magnetic resonance in medicine : MRM 2016, 75:196-206.

22. Wigström L, Ebbers T, Fyrenius a, Karlsson M, Engvall J, Wranne B, Bolger aF: Particle trace visualization of intracardiac flow using time-resolved 3D phase contrast MRI. Magnetic resonance in medicine : MRM 1999, 41:793-799.

23. Ebbers T HH, Dyverfeldt P, Sigfridsson A, Warntjes M, and Wigström L: Higher order weighted least-squares phase offset correction for improved accuracy in phase-contrast MRI. Abstracts, 16th Int Soc Magn Reson Med Toronto, 2008:1367. 
24. Dyverfeldt P, Sigfridsson A, Kvitting JP, Ebbers T: Quantification of intravoxel velocity standard deviation and turbulence intensity by generalizing phasecontrast MRI. Magnetic resonance in medicine : MRM 2006, 56:850-858.

25. Zajac J, Eriksson J, Dyverfeldt P, Bolger AF, Ebbers T, Carlhall CJ: Turbulent kinetic energy in normal and myopathic left ventricles. Journal of magnetic resonance imaging : JMRI 2014.

26. Heiberg E, Sjögren J, Ugander M, Carlsson M, Engblom H, Arheden H: Design and validation of Segment--freely available software for cardiovascular image analysis. BMC medical imaging 2010, 10:1.

27. Koo TK, Li MY: A Guideline of Selecting and Reporting Intraclass Correlation Coefficients for Reliability Research. J Chiropr Med 2016, 15:155-163.

28. El Rahman MYA, Abdul-Khaliq H, Vogel M, Alexi-Meskishvili V, Gutberlet M, Lange P: Relation between right ventricular enlargement, QRS duration, and right ventricular function in patients with tetralogy of Fallot and pulmonary regurgitation after surgical repair. Heart 2000, 84:416-420.

29. Baumgartner H, Bonhoeffer P, De Groot NM, de Haan F, Deanfield JE, Galie N, Gatzoulis MA, Gohlke-Baerwolf C, Kaemmerer H, Kilner P, et al: ESC Guidelines for the management of grown-up congenital heart disease (new version 2010). European heart journal 2010, 31:2915-2957.

30. Silversides CK, Marelli A, Beauchesne L, Dore A, Kiess M, Salehian O, Bradley T, Colman J, Connelly M, Harris L, et al: Canadian Cardiovascular Society 2009 Consensus Conference on the management of adults with congenital heart disease: executive summary. The Canadian journal of cardiology 2010, 26:143-150.

31. Warnes CA, Williams RG, Bashore TM, Child JS, Connolly HM, Dearani JA, del Nido P, Fasules JW, Graham TP, Jr., Hijazi ZM, et al: ACC/AHA 2008 guidelines 
for the management of adults with congenital heart disease: a report of the American College of Cardiology/American Heart Association Task Force on Practice Guidelines (Writing Committee to Develop Guidelines on the Management of Adults With Congenital Heart Disease). Journal of the American College of Cardiology 2008, 52:e143-263.

32. Jeong D, Anagnostopoulos PV, Roldan-Alzate A, Srinivasan S, Schiebler ML, Wieben O, Francois CJ: Ventricular kinetic energy may provide a novel noninvasive way to assess ventricular performance in patients with repaired tetralogy of Fallot. The Journal of thoracic and cardiovascular surgery 2015, 149:1339-1347.

33. Hirtler D, Garcia J, Barker AJ, Geiger J: Assessment of intracardiac flow and vorticity in the right heart of patients after repair of tetralogy of Fallot by flowsensitive 4D MRI. European radiology 2016.

34. Morales DL, Zafar F, Fraser CD: Tetralogy of Fallot repair: the Right Ventricle Infundibulum Sparing (RVIS) strategy. Seminars in thoracic and cardiovascular surgery Pediatric cardiac surgery annual 2009, 12:54-58.

35. Davlouros PA, Kilner PJ, Hornung TS, Li W, Francis JM, Moon JCC, Smith GC, Tat T, Pennell DJ, Gatzoulis MA: Right ventricular function in adults with repaired tetralogy of Fallot assessed with cardiovascular magnetic resonance imaging. Journal of the American College of Cardiology 2002, 40:2044-2052.

36. Frigiola A, Tsang V, Nordmeyer J, Lurz P, van Doorn C, Taylor AM, Bonhoeffer P, de Leval M: Current approaches to pulmonary regurgitation. European journal of cardio-thoracic surgery : official journal of the European Association for Cardiothoracic Surgery 2008, 34:576-580; discussion 581-572. 
37. Babu-Narayan SV, Kilner PJ, Li W, Moon JC, Goktekin O, Davlouros PA, Khan M, Ho SY, Pennell DJ, Gatzoulis MA: Ventricular fibrosis suggested by cardiovascular magnetic resonance in adults with repaired tetralogy of fallot and its relationship to adverse markers of clinical outcome. Circulation 2006, 113:405413.

38. Wald RM, Haber I, Wald R, Valente AM, Powell AJ, Geva T: Effects of regional dysfunction and late gadolinium enhancement on global right ventricular function and exercise capacity in patients with repaired tetralogy of Fallot. Circulation 2009, 119:1370-1377.

39. Oosterhof T, Mulder BJ, Vliegen HW, de Roos A: Corrected tetralogy of Fallot: delayed enhancement in right ventricular outflow tract. Radiology 2005, 237:868871.

40. Munkhammar $\mathrm{P}$, Carlsson $\mathrm{M}$, Arheden $\mathrm{H}$, Pesonen $\mathrm{E}$ : Restrictive right ventricular physiology after tetralogy of Fallot repair is associated with fibrosis of the right ventricular outflow tract visualized on cardiac magnetic resonance imaging. European heart journal cardiovascular Imaging 2013, 14:978-985.

41. Zile MR, Gregg D: Is Biventricular Fibrosis the Mediator of Late Complications in Tetralogy of Fallot? JACC Cardiovascular imaging 2016, 9:11-13.

42. Chen CA, Dusenbery SM, Valente AM, Powell AJ, Geva T: Myocardial ECV Fraction Assessed by CMR Is Associated With Type of Hemodynamic Load and Arrhythmia in Repaired Tetralogy of Fallot. JACC Cardiovascular imaging 2016, 9:1-10.

43. Dyverfeldt P, Ebbers T: Letter by Dyverfeldt and Ebbers regarding article "Estimation of turbulent kinetic energy using 4D phase-contrast MRI: Effect of 
scan parameters and target vessel size". Magnetic resonance imaging 2016, 34:1226. 


\section{Tables}

Table 1. ANOVA of demographic and clinical data for the three study groups.

\begin{tabular}{|c|c|c|c|}
\hline & $\begin{array}{l}\text { Healthy control group } \\
\qquad \mathrm{N}=10\end{array}$ & $\begin{array}{c}\text { Fallot with lower } \\
\text { PR fraction }(\leq 11 \%) \\
N=9\end{array}$ & $\begin{array}{c}\text { Fallot with higher } \\
\text { PR fraction }(>11 \%) \\
\text { N=8 }\end{array}$ \\
\hline Age (y) & $\begin{array}{c}31 \pm 11 \\
(22-54)\end{array}$ & $\begin{array}{c}30 \pm 8 \\
(21-47)\end{array}$ & $\begin{array}{c}36 \pm 14 \\
(23-65)\end{array}$ \\
\hline Body Surface Area $\left(\mathrm{m}^{2}\right)$ & $\begin{array}{c}1.94 \pm 0.21 \\
(1.60-2.37)\end{array}$ & $\begin{array}{c}1.94 \pm 0.21 \\
(1.69-2.24)\end{array}$ & $\begin{array}{c}1.85 \pm 0.27 \\
(1.46-2.33)\end{array}$ \\
\hline $\mathrm{HR}$ at rest (bpm) & $\begin{array}{l}70 \pm 10 \\
(55-87)\end{array}$ & $\begin{array}{c}76 \pm 12 \\
(61-102)\end{array}$ & $\begin{array}{c}70 \pm 13 \\
(54-89)\end{array}$ \\
\hline Systolic blood pressure (mmHg) & $\begin{array}{c}126 \pm 10 \\
(115-150)\end{array}$ & $\begin{array}{c}119 \pm 16 \\
(95-140)\end{array}$ & $\begin{array}{c}119 \pm 13 \\
(95-135)\end{array}$ \\
\hline Diastolic blood pressure (mmHg) & $\begin{array}{c}75 \pm 7 \\
(60-80)\end{array}$ & $\begin{array}{c}75 \pm 12 \\
(60-90)\end{array}$ & $\begin{array}{c}73 \pm 13 \\
(55-90)\end{array}$ \\
\hline RVEDVI (ml/m²) & $\begin{array}{c}93 \pm 15 \\
(70-118)\end{array}$ & $\begin{array}{c}107 \pm 22 \\
(75-145)\end{array}$ & $\begin{array}{c}140 \pm 25^{* * *+\dagger} \\
(103-179)\end{array}$ \\
\hline RVESVI (ml/m²) & $\begin{array}{c}39 \pm 8 \\
(22-48)\end{array}$ & $\begin{array}{l}58 \pm 12^{* *} \\
(46-77)\end{array}$ & $\begin{array}{c}82 \pm 15^{* * *+\dagger \dagger \dagger} \\
(59-100)\end{array}$ \\
\hline RVEF (\%) & $\begin{array}{c}58 \pm 7 \\
(48-69)\end{array}$ & $\begin{array}{l}46 \pm 5^{* * *} \\
(38-54)\end{array}$ & $\begin{array}{c}42 \pm 4^{* * *} \\
(38-48)\end{array}$ \\
\hline QRS-duration (ms) & - & $\begin{array}{c}134 \pm 10 \\
(82-176)\end{array}$ & $\begin{array}{c}156 \pm 5 \\
(132-182)\end{array}$ \\
\hline Time from ToF repair $(y)$ & - & $\begin{array}{c}25 \pm 9 \\
(8-40)\end{array}$ & $\begin{array}{c}32 \pm 9 \\
(22-51)\end{array}$ \\
\hline
\end{tabular}

${ }^{* *} \mathrm{p}<0.01$ vs Healthy control group, $* * * \mathrm{p}<0.001$ vs Healthy control group.

$\dagger \dagger p<0.01$ vs Fallot with lower PR fraction $(\leq 11 \%), \dagger \dagger \dagger p<0.001$ vs Fallot with lower PR fraction $(\leq 11 \%)$.

$\mathrm{HR}=$ heart rate, $\mathrm{PR}=$ pulmonary regurgitation, $\mathrm{RVEDVI}=$ right ventricular end diastolic volume indexed, RVESVI $=$ right ventricular end systolic volume indexed, RVEF = right ventricular ejection fraction, ToF = tetralogy of Fallot. 
Table 2. ANOVA of conventional 2D flow and 4D flow specific parameters for the three study groups.

\begin{tabular}{|c|c|c|c|}
\hline Conventional $2 D$ flow parameters & $\begin{array}{l}\text { Healthy control group } \\
\qquad \mathrm{N}=9\end{array}$ & $\begin{array}{c}\text { Fallot with lower } \\
\text { PR fraction }(\leq 11 \%) \\
\text { N=9 }\end{array}$ & $\begin{array}{c}\text { Fallot with higher } \\
\text { PR fraction }(>11 \%) \\
\text { N=8 }\end{array}$ \\
\hline PR Volume (ml) & $\begin{array}{c}1.5 \pm 0.9 \\
(0.4-3.2)\end{array}$ & $\begin{array}{c}3.9 \pm 3.7 \\
(0.4-11)\end{array}$ & $\begin{array}{c}37 \pm 20 * * * \dagger \dagger \dagger \\
(14-67)\end{array}$ \\
\hline PR Fraction (\%) & $\begin{array}{l}2 \pm 0.7 \\
(1-3)\end{array}$ & $\begin{array}{c}4 \pm 4 \\
(1-11)\end{array}$ & $\begin{array}{c}32 \pm 11^{* * *+\dagger \dagger} \\
(16-50)\end{array}$ \\
\hline 4D flow specific parameters & $\begin{array}{l}\text { Healthy control group } \\
\qquad \mathrm{N}=10\end{array}$ & $\begin{array}{c}\text { Fallot with lower } \\
\text { PR fraction }(\leq 11 \%) \\
\text { N=9 }\end{array}$ & $\begin{array}{l}\text { Fallot with higher } \\
\text { PR fraction }(>11 \%) \\
\quad \mathrm{N}=8\end{array}$ \\
\hline Average RV TKE (mJ) & $\begin{array}{c}1.24 \pm 0.53 \\
(0.63-2.16)\end{array}$ & $\begin{array}{c}1.44 \pm 0.49 \\
(1.00-2.26)\end{array}$ & $\begin{array}{c}3.22 \pm 1.58 * * * \dagger \dagger \\
(1.18-4.87)\end{array}$ \\
\hline Peak Total RV TKE (mJ) & $\begin{array}{c}1.91 \pm 0.78 \\
(0.91-3.33)\end{array}$ & $\begin{array}{c}2.23 \pm 0.81 \\
(1.47-3.74)\end{array}$ & $\begin{array}{l}5.95 \pm 3.15 * * * \dagger \dagger \\
\quad(2.26-10.6)\end{array}$ \\
\hline Peak RV TKE (mJ/m³) & $\begin{array}{c}86.3 \pm 22.1 \\
(54.7-112) \\
\end{array}$ & $\begin{array}{c}130 \pm 70.6 \\
(58.7-246) \\
\end{array}$ & $\begin{array}{c}343 \pm 71.5^{* * * * \dagger \dagger \dagger} \\
(263-494) \\
\end{array}$ \\
\hline
\end{tabular}

${ }^{*} \mathrm{p}<0.05$ vs Healthy control group, ${ }^{* *} \mathrm{p}<0.01$ vs Healthy control group, ${ }^{* * *} \mathrm{p}<0.001$ vs Healthy control group. $\dagger \dagger \mathrm{p}<0.01$ vs Fallot with lower PR fraction $(\leq 11 \%), \dagger \dagger \dagger \mathrm{p}<0.001$ vs Fallot with lower PR fraction $(\leq 11 \%)$.

$\mathrm{PR}=$ pulmonary regurgitation, $\mathrm{TKE}=$ turbulent kinetic energy. 
1 Table 3. Simple and multiple regression analyses for parameters against RVEDVI.

\begin{tabular}{|c|c|c|c|c|c|c|}
\hline \multicolumn{7}{|c|}{ Multiple regression model for parameters against RVEDVI $\left(\mathrm{ml} / \mathrm{m}^{3}\right)(\mathrm{N}=17)$} \\
\hline Parameter tested & $\begin{array}{l}\text { Simple regression, } \\
P \text {-values } \\
R^{2} \text {-values }\end{array}$ & $\begin{array}{l}\text { Multiple regression } \\
\text { step 1,P-values }\end{array}$ & $\begin{array}{l}\text { Multiple regression } \\
\text { step 2, } P \text {-values }\end{array}$ & $\begin{array}{l}\text { Multiple regression } \\
\text { step 3, P-values }\end{array}$ & $\begin{array}{l}\text { Multiple regression } \\
\text { step 4, P-values }\end{array}$ & $\begin{array}{l}\text { Multiple regression } \\
\text { step 5, P-values }\end{array}$ \\
\hline \multicolumn{7}{|l|}{ Clinical parameters } \\
\hline \multirow[t]{2}{*}{ Age $(y)$} & $\mathrm{p}=0.840$ & Out & & & & \\
\hline & $\mathrm{R}^{2}=0.003$ & & & & & \\
\hline \multirow[t]{2}{*}{ Body Surface Area $\left(m^{2}\right)$} & $\mathrm{p}=0.490$ & Out & & & & \\
\hline & $\mathrm{R}^{2}=0.032$ & & & & & \\
\hline \multirow[t]{2}{*}{ Time from ToF repair (y) } & $\mathrm{p}=0.912$ & Out & & & & \\
\hline & $\mathrm{R}^{2}=0.001$ & & & & & \\
\hline \multicolumn{7}{|l|}{ Conventional parameters } \\
\hline \multirow[t]{2}{*}{ PR Volume (ml) } & $\mathrm{p}=0.009^{*}$ & $\mathrm{p}=0.272$ & $\mathrm{p}=0.243$ & $\mathrm{p}=0.301$ & $\mathrm{p}=0.603$ & Out \\
\hline & $\mathbf{R}^{2}=\mathbf{0 . 3 7}$ & & & & & \\
\hline \multirow[t]{2}{*}{ PR Fraction (\%) } & $\mathrm{p}=0.013 *$ & $\mathrm{p}=0.411$ & $\mathrm{p}=0.292$ & $\mathrm{p}=0.365$ & Out & \\
\hline & $\mathbf{R}^{2}=0.35$ & & & & & \\
\hline \multicolumn{7}{|l|}{ 4D flow specific parameters } \\
\hline \multirow[t]{2}{*}{ Average RV TKE $(\mathrm{mJ} / \mathrm{ml})$} & $\mathrm{p}=0.004 *$ & $\mathrm{p}=0.573$ & $\mathrm{p}=0.534$ & Out & & \\
\hline & $\mathbf{R}^{2}=\mathbf{0 . 4 4}$ & & & & & \\
\hline \multirow[t]{2}{*}{ Peak Total RV TKE (mJ/ml) } & $\mathrm{p}=0.002 *$ & $\mathrm{p}=0.269$ & $\mathrm{p}=0.247$ & $\mathrm{p}=0.069$ & $\mathrm{p}=0.101$ & $\mathrm{p}=0.002^{*}$ \\
\hline & $\mathbf{R}^{2}=0.47$ & & & & & \\
\hline \multirow[t]{2}{*}{ Peak RV TKE $\left(\mathrm{mJ} / \mathrm{m}^{3}\right)$} & $\mathrm{p}=0.039 *$ & $\mathrm{p}=0.919$ & Out & & & \\
\hline & $\mathrm{R}^{2}=0.25$ & & & & & \\
\hline $\mathrm{R}^{2}$ for model & & $\mathrm{R}^{2}=0.53$ & $\mathrm{R}^{2}=0.53$ & $\mathrm{R}^{2}=0.52$ & $\mathrm{R}^{2}=0.48$ & $\mathrm{R}^{2}=0.47$ \\
\hline Adjusted $\mathrm{R}^{2}$ for model & & $\mathrm{R}^{2}{ }_{\text {adj }}=0.32$ & $\mathrm{R}^{2}{ }_{\mathrm{adj}}=0.38$ & $\mathrm{R}^{2}{ }_{\mathrm{adj}}=0.41$ & $\mathrm{R}^{2}{ }_{\text {adj }}=0.41$ & $\mathbf{R}^{2}{ }_{\text {adj }}=\mathbf{0 . 4 4}$ \\
\hline p-value for model & & $\mathrm{p}=0.094$ & $\mathbf{p}=0.044^{*}$ & $\mathbf{p}=0.02 *$ & $\mathbf{p}=0.01 *$ & $\mathbf{p}=0.002 *$ \\
\hline
\end{tabular}

$\mathrm{PR}=$ pulmonary regurgitation, RVEDVI = right ventricular end diastolic volume indexed, RVEF = right ventricular ejection fraction, ToF $=$ tetralogy of Fallot 


\section{Figure legends}

2 Figure 1. Visualization of turbulent kinetic energy (TKE) in the RV of an early repair

3 Tetralogy of Fallot patient with PR fraction 30\% in three progressive diastolic time frames. At

4 early diastolic filling, blood starts to flow from the right atrium (RA) through the tricuspid

5 valve (TV) (A). The regurgitant blood (white arrow) from the pulmonary circulation (Pulm)

6 enters through the insufficient pulmonary valve (PV) (B and C). Adjacent to the regurgitant

7 flow, development of TKE is evident by the yellow-red plume (white arrow) (A through C).

8 Higher levels of TKE are represented as bright yellow and lower levels as increasingly

9 reddish. Peak $R V$ TKE is shown graphically over diastole with a line marking the respective

10 time frame of diastole (A through C). The anatomic position of the Peak $R V T K E$ value in the

11 respective time frame is shown as a green dot, present centrally in the TKE plume. The RV is

12 visualized as a semi-transparent mask, and a four-chamber morphologic image provides

13 anatomic orientation.

Figure 2. TKE in the RV over diastole: A. in the healthy control group; B. in the Fallot group with lower PR fraction $(\leq 11 \%)$. C. in the Fallot group with higher PR fraction $(>11 \%)$. No significant difference was found between lower PR fraction group and the healthy control group, illustrated by the graphs A and B (Table 2). The graph C illustrates the clearly visible higher TKE levels of patients with the highest PR fractions: 50\% (a), 39\% (b), 40\% (c), 30\% (d) and 28\% (e), respectively.

Figure 3. Linear regression between Peak Total RV TKE and RVEDVI for the patient cohort $(\mathrm{N}=17)$. The lower and higher $\mathrm{PR}$ fraction groups are represented by triangles and dots, respectively. 


\section{Supporting information}

3 Supporting file 1, movie. Visualization of turbulent kinetic energy (TKE) throughout

4 diastole in the right ventricle (RV) of a Tetralogy of Fallot patient with total repair including

5 transannular patching and PR fraction $3 \%$. In the RV outflow tract, the development of TKE

6 around the subtle pulmonary regurgitation is portrayed by the small yellow-red plume. Higher

7 levels of TKE are represented as bright yellow and lower levels as increasingly reddish. The

$8 \quad \mathrm{RV}$ is visualized as a semi-transparent mask, and a four-chamber morphologic image provides

9 anatomic orientation. Please see Supporting file 2 for comparison.

11 Supporting file 2, movie. Visualization of turbulent kinetic energy (TKE) throughout 12 diastole in the right ventricle (RV) of a Tetralogy of Fallot patient with initial Blalock13 Taussig shunt, subsequent total repair including transannular patching and PR fraction $40 \%$. 14 In the RV outflow tract, the development of TKE around the significant PR is evident by the 15 large yellow-red plume, reaching centrally into the RV. Higher levels of TKE are represented as bright yellow and lower levels as increasingly reddish. The RV is visualized as a semi-

17 transparent mask, and a four-chamber morphologic image provides anatomic orientation.

18 Please see Supporting file 1 for comparison. 


\section{$A$}

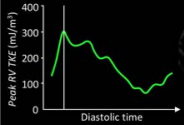

Pulm

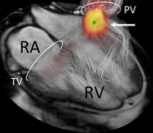

B
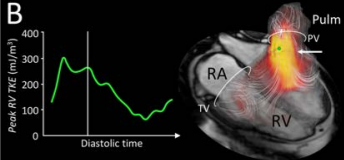

C
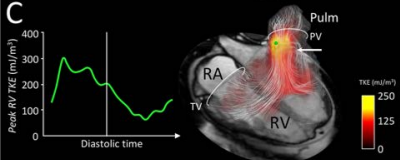

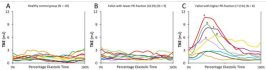


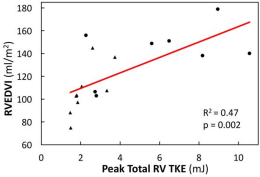

